\title{
Targeted Therapy For AIDS-related Kaposi's Sarcoma Henry Koon*‡
}

\author{
Address: Beth Israel Deaconess Medical Center/Harvard Medical School, Boston, MA \\ Email: Henry Koon* - hkoon@bidmc.harvard.edu \\ * Corresponding author ‡Presenting author
}

from 2005 International Meeting of The Institute of Human Virology

Baltimore, USA, 29 August - 2 September 2005

Published: 8 December 2005

Retrovirology 2005, 2(SuppI I):S45 doi:I0.I 186/1742-4690-2-SI-S45

Angiogenesis plays a critical in the pathogenesis of Kaposi's sarcoma. Platelet derived growth factor (PDGF), vascular endothelial growth factor, fibroblast growth factor and matrix metalloproteinases (MMP) are among the angiogenic pathways that have been implicated in the development of Kaposi's sarcoma. The introduction of specific therapies such as imatinib and Col-3 that target the PDGF pathway and MMPs, respectively, provide a mechanism to test the importance of these pathways in Kaposi's sarcoma in vivo. In this session the rationale for targeted therapy in Kaposi's sarcoma will be discussed. The results from recent trials involving anti-angiogenic agents and signal transduction inhibitors will be reviewed. Additionally, trials of targeted therapies that are underway or in development will be outlined. 